\title{
REQUIREMENTS OPTIMIZATION FOR SMALL SATEllite OPTICAL COMMUNiCATiONS
}

\author{
STEPANOVA, D. \& PRYANICHNIKOV, V.
}

Abstract: An analytical approach to define requirements set for free-space optical communications for small satellites is considered. Several scenarios are reviewed: intersatellite link and satellite to ground communication. Equations describing satellite orbital state vector, orientation, power and link budgets are defined. An optimal satellite setup is derived from the optimization problem: the maximum communication speed is subjected to minimum of power consumption.

Key words: Satellite optical communication, Control system, CubeSat, Free-space optical communication, pointing and tracking system.
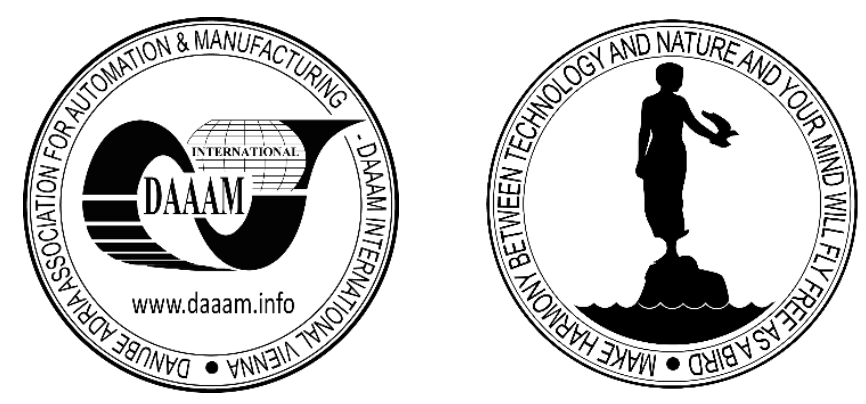

Authors' data: Dipl.-Ing. PhD student Stepanova, D[aria]*; Univ.Prof. Dipl.-Ing. Pryanichnikov, V[alentin]**, * Moscow Institute of Physics and Technology, 9 Institutskiy per., Dolgoprudny, Moscow Region, 141701, Russian Federation, ** KIAM Russian Academy of Sciences, MSTU "Stankin" and IINET RSUH, Moscow 127055, Russia, stepanova.d@phystech.edu, v.e.pr@yandex.ru

This Publication has to be referred as: Stepanova, D[aria] \& Pryanichnikov, V[alentin] (2019). Requirements Optimization for Small Satellite Optical Communications, Chapter 28 in DAAAM International Scientific Book 2019, pp.331-342, B. Katalinic (Ed.), Published by DAAAM International, ISBN 978-3902734-24-2, ISSN 1726-9687, Vienna, Austria

DOI: $10.2507 /$ daaam.scibook.2019.28 
Stepanova, D. \& Pryanichnikov, V.: Requirements Optimization for Small Satellite ...

\section{Introduction}

In the past decade, small satellites and CubeSats in particular became a new trend in the aerospace industry. So far, CubeSats occupied the technology demonstration sector, university level missions and partially missions for Earth Observation. While technology was maturing, some applications in the field of Internet of Things, mobile platforms tracking and advanced imagery acquisition also became relevant. It can be observed, that with advancement of payloads and missions, the demand for data downlink from the satellite to the ground is rapidly growing, thus posing new requirements on transceivers for higher data downlink rates. Besides that, in new applications involving intersatellite link establishment, an alternative to radio frequency communication might solve the critical problem of radio frequency coordination. Optical communication is an advantageous alternative to excising solutions: it requires smaller terminal and antenna size, less operational power, higher bandwidth and as the result, higher transmission rates.

There are two scenarios of optical link utilisation on a small satellite: intersatellite connection or satellite to ground link. Both of the scenarios pose strict limitations on the optical terminal design as well as the satellite setup. The satellite system influenced the most by these requirements is an Attitude Determination and Control System (ADCS): the dependence of the output power and laser beam divergence is proportional to the satellite pointing capabilities. Besides that, optical terminals might incorporate an internal Pointing, Acquisition and Tracking (PAT) system in order to compensate the satellite jitter and initial pointing error. Development of such system is also a challenge.

There are several concepts for optical terminal design for small satellites (Kaymak et al., 2018): Gimbal-Based mechanisms, Mirror-Based mechanisms, Gimbal-Mirror Hybrid mechanisms, Adaptive Optics, Liquid-Crystal-Based Mechanisms. Besides that, small satellites, benefitting from the body size, consider body-pointing approach without any additional hardware. Several concepts have already been successfully validated on the orbit (Janson et al., 2014, August), (Rose et al., 2019), (Clements et al., 2016) and several missions are currently under development (Iwamoto et al., 2017), (Stepanova et al., 2018).

However, based on NASA CubeSat state of the art report (Sst-soa.arc.nasa.gov. 2019), the technology readiness level of a CubeSat based optical communication is still on the level 7. Major challenges preventing the technology from further development are pointing capabilities influenced by satellite errors, vibrations and noises, large field of views limited by the telescope capabilities. Existing laser terminal and satellite solutions are constrained by the balance between satellite mass, power requirements, telescope complexity.

Current work is dedicated to the requirements analysis for the satellite optical communication terminal development and a satellite itself. The main idea of the work is do define the general mathematical model of the satellite and optical terminal and find an optimal configuration from power, dynamics and communication speed point of view. For that, the optimization problem is introduced: maximization of transmission speed subject to maintaining link, mass and power budgets. 


\section{Problem statement}

The goal of current work is to find the optimal satellite configuration for optical communications establishment in different scenarios. In order to define optimisation problem, following assumptions are made.

- Small satellites of the CubeSat form factor from $1 \mathrm{U}$ till $16 \mathrm{U}$ are reviewed.

- Low Earth Orbit (LEO) is the most attractive orbit for CubeSats due to amount of rideshare opportunities. Thus, LEO from 400 to $650 \mathrm{~km}$ is considered based on the historical launch data.

- Most of CubeSats are launched to the polar orbit with inclination of $98 \mathrm{deg}$.

- In order to simplify the model definition, it is assumed, that the satellite and PAT system are symmetrical about the main axes of inertia and the center of mass of the satellite lies in the geometric center

- The standard unit mass of a CubeSat requirement is not strict and normally depends on the launch provider agreement. Based on historical data, an average mass of $1 \mathrm{U}$ is around $1.52 \mathrm{~kg}$. For further simplification, we assume, that mass of the satellite is proportional to its size.

- To simplify the model, one ground station is available for data downlink. As no specific mission is defined, one ground station will be sufficient to derive optimal requirements set.

- Communication with the ground station is limited by elevation angles of 20 degrees. Below these angles, loses due to the large distances and atmosphere are considerable.

- Bit error rate considerably depends on the type of modulation. For the current work, On-Off Keying modulation is chosen, as the most common for freespace optical communications.

- Pointing system is presented by a gimbal mechanism. Derived model can also be used for the piezo driver integrated in the telescope system.

There are two scenarios covered in the current work: inter-satellite communications and satellite-ground communications. First scenario is limited by two cases: satellites are tracking each other on the same orbit and satellites passing each other with different angles of perigee. In first case, the relative angular velocity remains constant, the second case will require rapid slew rates during passes.

Input parameters for the satellite mathematical model are set as input arrays with defined steps.

0 The difference between orbits in first scenario varies from 0 to 180 degrees with step of 5 degrees. In second scenario, satellite passes the ground station and relative angular rates remain average. This scenario will be influenced by the maximum satellite elevation and azimuth angles, which can change from 20 to 160 degrees both.

- Orbit altitude changes from 400 to $650 \mathrm{~km}$ with step $10 \mathrm{~km}$.

- Satellites from factors vary from $1 \mathrm{U}, 2 \mathrm{U}, 3 \mathrm{U}, 6 \mathrm{U}$, and $12 \mathrm{U}$ till $16 \mathrm{U}$.

- For the satellite stabilisation different instrumentation might be used. First solution is based on 3-axes reaction wheels, while another option is Fluid 
Stepanova, D. \& Pryanichnikov, V.: Requirements Optimization for Small Satellite ...

Dynamic Actuator (FDA). Each of systems is defined with following parameters: maximum torque, maximum angular momentum, power consumption, momentum storage, and balance characteristics.

Design parameters are defined using the simulation model and are used to track the system limitations. These parameters are also later fed to the optimization function.

- Relative angular velocities are design drivers. Depending on the chosen scenarios, they affect the attitude control manoeuvres and, thus, choice of actuators.

- Tracking speed limitations are also set by the choice of actuators and, thus, satellite capabilities.

- Power consumption of the satellite bus and payload is assumed to be proportional to the size: the bigger the satellite, the more power-demanding payload it can accommodate. Power input is set to an orbit average for a defined satellite surface area.

- Accuracy of the orientation determination system is based on the sensors set implemented on the spacecraft. Based on historical data, accuracy of the orientation determination varies with satellite size and complexity. For example, $1 \mathrm{U}$ CubeSat is not likely to carry the star tracker and thus attitude knowledge will be much less than $3 \mathrm{U}$ CubeSat might have. For the current model, attitude knowledge is fixed to the size of the satellite.

\section{Satellite modelling}

In order to simulate the performance of the whole system, dynamic model of the satellite, link budget estimation and power balance calculations are used (Cui \& Yan, 2012), (Ivanov et al., 2015), (Abdo et al., 2013), (Li et al., 2014).

To derive the dynamic equation of the satellite setup we need first introduce the coordinate frames. Following coordinate frames will be used: Earth inertia frame $\mathrm{Ox}_{\mathrm{i}} \mathrm{y}_{\mathrm{i}} \mathrm{Z}_{\mathrm{i}}$, Orbit frame with satellite mass center in the origin of the frame $\mathrm{Ox}_{0} \mathrm{y}_{0} \mathrm{z}_{0}$, Satellite body frame Oxyz, pointing system frame OхРАтУратZрАт, which is connected to the optical transceiver, and a gimbal frame $\mathrm{Ox}_{\mathrm{G}} \mathrm{y}_{\mathrm{G}} \mathrm{Z}_{\mathrm{G}}$. Fig.1 and 2 represent the frames of the satellite and laser terminal.

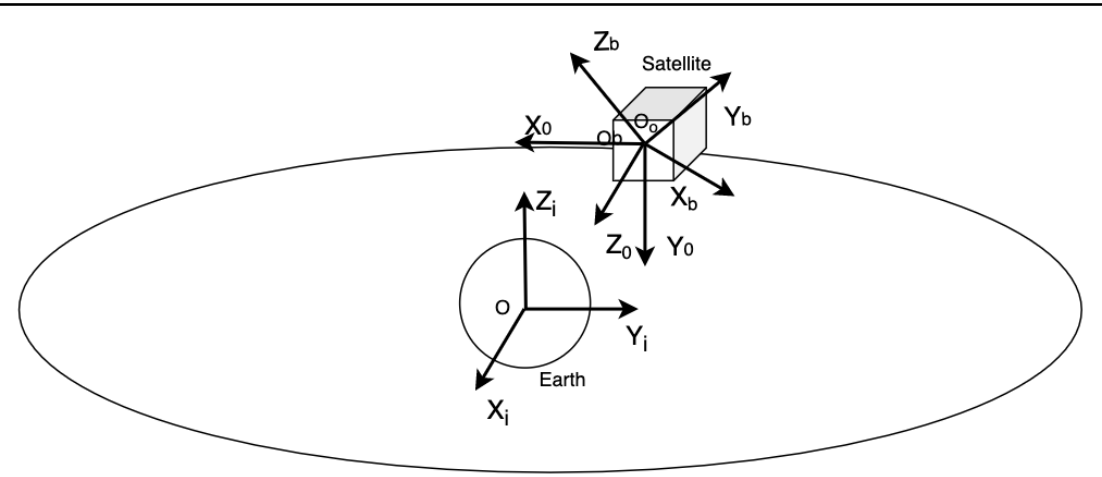

Fig. 1. Satellite and Earth coordinate frames. 


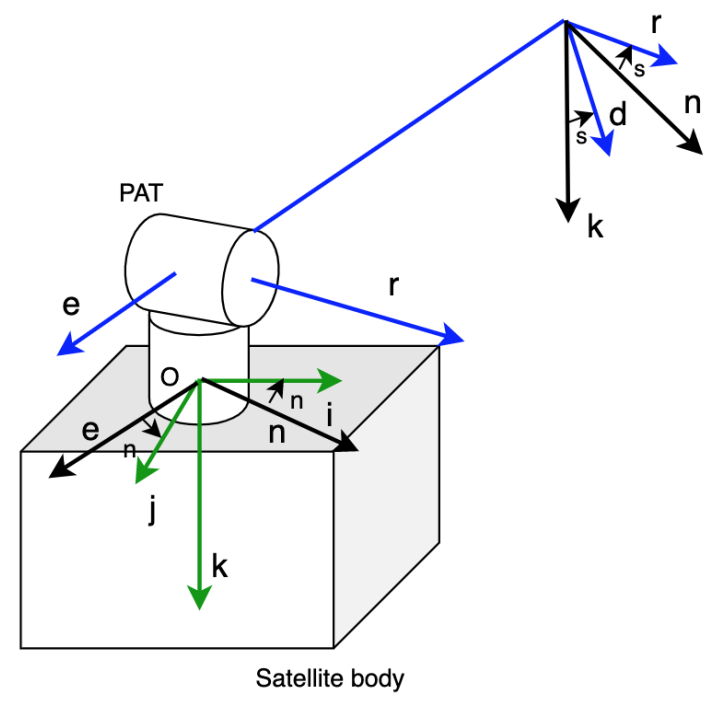

Fig. 2. Pointing systems of laser terminal coordinate frames.

In the gimbal frame dynamic model of the PAT system can be defined from Euler equation:

$$
M_{P A T}=K_{P A T}^{\prime}+\omega_{P A T}^{a} \times K_{P A T}
$$

where $M_{P A T}$ - external torques; $K_{P A T}$ - angular momentum acting on the PAT drivers; $\omega_{\text {PAT }}^{a}$ - absolute PAT angular velocity in gimbal frame. $K_{P A T}$ is defined with inertia tensor and angular speed:

$$
\begin{aligned}
K_{P A T} & =J_{P A T} \omega_{\mathrm{PAT}}^{a} \\
J_{P A T} & =\left[\begin{array}{ccc}
\mathrm{J}_{x x} & 0 & 0 \\
0 & \mathrm{yy} & 0 \\
0 & 0 & \mathrm{~J}_{z z}
\end{array}\right]
\end{aligned}
$$

$\mathrm{J}_{x x}, \mathrm{~J}_{y y}, \mathrm{~J}_{z z}$ - moments of inertia around axes $\mathrm{x}, \mathrm{y}, \mathrm{z}$. Here we assume, that center of inertia coincides with geometrical center of the pointing system.

Three forces act on the satellite: gravitational, magnetic field, plus the moment of the orientation system. We assume that the satellite is a solid body moving in a circumcircular orbit around the Earth; the SGP4 model is used to describe the Earth's gravitational field. The IGRF model describes the geomagnetic field.

The change in satellite orientation and speed is described using the dynamic Euler equations and kinematic relationships based on quaternions, matrix elements of the direction cosines or Euler angles. The satellite state vector is described using angular velocity components and various sets of positional variables presented by quaternions $\Lambda=\left(q, q_{0}\right)$. Dynamic model (Golubev, 2000) taking into account the work of the orientation system is defined as: 
Stepanova, D. \& Pryanichnikov, V.: Requirements Optimization for Small Satellite ...

$$
\begin{gathered}
\frac{d J_{\text {sat }} \Omega}{d t}+\frac{d K_{P A T}}{d t}+\frac{d K_{A D C S}}{d t}+\Omega \times\left(J_{\text {sat }} \Omega+K_{P A T}+K_{R W}\right) \\
=M_{\text {mag }}+M_{\text {grav }}
\end{gathered}
$$

$\Omega$ - Satellite absolute angular velocity, $J_{\text {sat }}$ - satellite moment of inertia, $M_{\text {mag }}, M_{\text {grav }}$ are the magnetic moment, gravitational moment, $K_{P A T}, K_{A D C S}$ angular momentum of mechanical moment from the stabilization system and PAT system. External moments can be expressed as:

$$
M_{\text {grav }}=\frac{3 \mu}{R^{3}}(\mathrm{n} \times J \mathrm{n})
$$

$R$ - radius vector from Earth center to the satellite center of mass, $n=$ $\left[\begin{array}{lll}n_{x} & n_{y} & n_{z}\end{array}\right]^{T}$ - the vector of the local vertical in the satellite coordinate system, $\mu$ is the Earth gravitational constant. Magnetic moment is defined as:

$$
M_{m a g}=m \times B
$$

$m$ is the dipole moment of a satellite, B - vector of geomagnetic induction. $K_{A D C S}$ is a momentum from ADCS of a satellite, $\frac{\mathrm{d} K_{A D C S}}{d t}$ is ADCS control torque which is determined by

$$
\frac{\mathrm{d} K_{A D C S}}{d t}=K_{a} \lambda_{r e f}+K_{\omega}\left(\Omega-\Omega_{0}\right)-\Omega \times\left(J_{s a t} \Omega+K_{A D C S}\right)
$$

$\lambda_{r e f}$ is a vector part of the quaternion

$$
\Lambda_{\text {rel }}=\left[\begin{array}{ll}
\lambda_{\text {ref }}^{T} & \lambda_{\text {ref }}^{0}
\end{array}\right]^{T}=\Lambda_{0}^{-1} \Lambda
$$

Quaternion $\Lambda=\left[\begin{array}{ll}\lambda^{T} & \lambda^{0}\end{array}\right]^{T}$ sets the transition from the local-vertical-localhorizontal (LVLH) frame to the body-fixed frame, $\Lambda_{0}$ is required orientation, $\Omega$ is a vector of angular velocity of body-fixed frame with respect to LVLH, $\Omega_{0}$ - is a desired angular velocity, $K_{a}=\operatorname{diag}\left(K_{a}^{1}, K_{a}^{2}, K_{a}^{3}\right)$ and $K_{\omega}=\operatorname{diag}\left(K_{\omega}^{1}, K_{\omega}^{2}, K_{\omega}^{3}\right)$, are diagonal matrices of control parameters of ADCS.

Kinematic relations supplement dynamic equations. In numerical modeling, a quaternion is used to describe the satellite's orientation and the kinematic relation

$$
\Lambda=\frac{1}{2} C \Lambda
$$




$$
C=\left[\begin{array}{cccc}
0 & \Omega_{3} & -\Omega_{2} & \Omega_{1} \\
-\Omega_{3} & 0 & \Omega_{1} & \Omega_{2} \\
\Omega_{2} & -\Omega_{1} & 0 & \Omega_{3} \\
-\Omega_{1} & -\Omega_{2} & -\Omega_{3} & 0
\end{array}\right]
$$

Satellite tensor of inertia is defined as:

$$
J=\frac{m}{12}\left[\begin{array}{ccc}
(b+c)^{2} & 0 & 0 \\
0 & (a+c)^{2} & 0 \\
0 & 0 & (a+b)^{2}
\end{array}\right]
$$

To connect the dynamic model of the satellite with laser terminal parameters other dependences shall be introduced. Assuming that $1 \mathrm{U}$ CubeSat occupies $1.52 \mathrm{~kg}$ per 1U, satellite mass on size dependence can estimated as

$$
m=1.52 * a b c
$$

a, b, c - satellite parameters (Fig.3). Solar panels surface area and satellite size are connected as:

$$
S=2 a b+2 a c+b c
$$

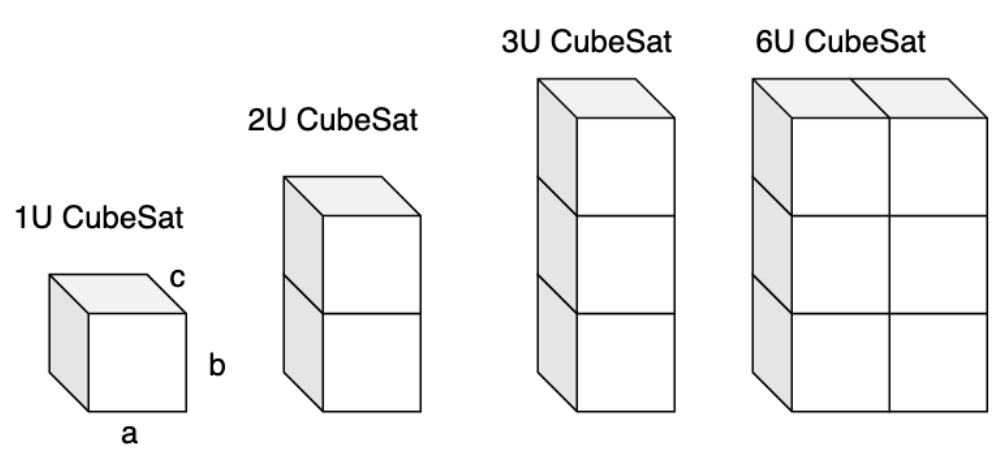

Fig. 3. Different CubeSat sizes

The beam divergence $\varphi$ linearly depends on the pointing accuracy. It shall be at least twice bigger than the accuracy in order to cover the partner for communication with laser spot. Fig.4 represents the dependence: angle a - angle between vector ground station to satellite and satellite pointing vector.

$$
\varphi=2 a
$$

In order to determine the maximum transmission rate, it is necessary to detect the intensity of the received radiation power. To do this, we compose the balance equation of the transmission line: 
Stepanova, D. \& Pryanichnikov, V.: Requirements Optimization for Small Satellite ...

$$
P_{\text {in }}=P_{\text {out }}+G_{r}+G_{t}-L
$$

$P_{\text {in }}$ - power at the receiver antenna, $P_{\text {out }}$ - transmission power, $G_{r}$ - gain of receiver antenna, $G_{t}$ - gate of the transmitter antenna, $L$ - channel losses.

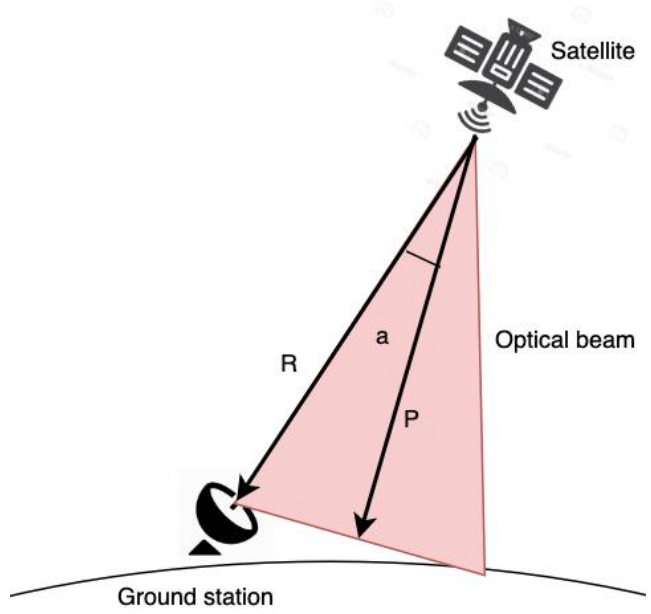

Fig. 4. Beam divergence dependence on satellite pointing accuracy

The transmission speed depends on the bandwidth, received power and modulation type. The simplest and most common modulation scheme for optical communication is On-Off Keying (OOK). Energy per bit to noise power spectral density ratio (EbNo) threshold can be calculated as

$$
\begin{aligned}
& E_{b} / N_{o t h}=E_{b} / N_{o_{s y s}}-R \\
& E_{b} / N_{o_{s y s}}=P_{\text {in }}-L_{G S}-B-G / T
\end{aligned}
$$

$R$ - Transmission rate in $\mathrm{dBHz}, G / T$ - receiver performance, $E_{b} / N_{o_{s y s}}$ system energy per bit to noise power spectral density ratio, $L_{G S}$ - Spacecraft Antenna Pointing Loss, $B$ - Boltzmann's Constant.

It is assumed, that satellite payload requires $50 \%$ from satellite average power generation. This number is derived from CubeSat historical data. Thus, power margin of the satellite is defined as:

$$
\mathrm{P}_{\text {marg }}=P_{\text {in }}-P_{P l}-P_{B}-P_{L T}
$$

$P_{i n}$ - input satellite power, $P_{P l^{-}}$power consumption of the payload, $P_{B}$ - bus power consumption, $P_{L T}$ - laser terminal power consumption.

As power, communication and orientation models of a satellite are defined; we can introduce the optimization problem to determine the optimal satellite setup. For each of defined scenarios, optimization problem is formulated as following. Maximize the objective function: 


$$
\mathrm{f}\left(t, \overline{\mathrm{r}_{\text {sat }}}, \overline{\omega_{P A T}}\right)=\left\{\begin{array}{c}
\text { Power }_{\text {margine }}\left(t, \overline{\mathrm{r}_{\text {sat }}}, \overline{\omega_{P A T}}\right) \\
R\left(t, \overline{\mathrm{r}_{\text {sat }}}, \overline{\omega_{P A T}}\right)
\end{array}\right.
$$

subject to

$$
\left\{\begin{array}{c}
1 U<\text { Size }<16 U \\
400<\text { Orbit }<650 \\
\text { ADCS constraints } \\
\text { Comm link constraints }
\end{array}\right.
$$

Defined optimization problem is solved using the Simplex method.

\section{Simulation results}

Models of satellite were realized in Matlab. Fig.4 represents results of orbital and dynamic simulation for three satellites in two scenarios (link between satellites and link between satellite and ground station). Blue, black and green areas correspond to communication sessions.

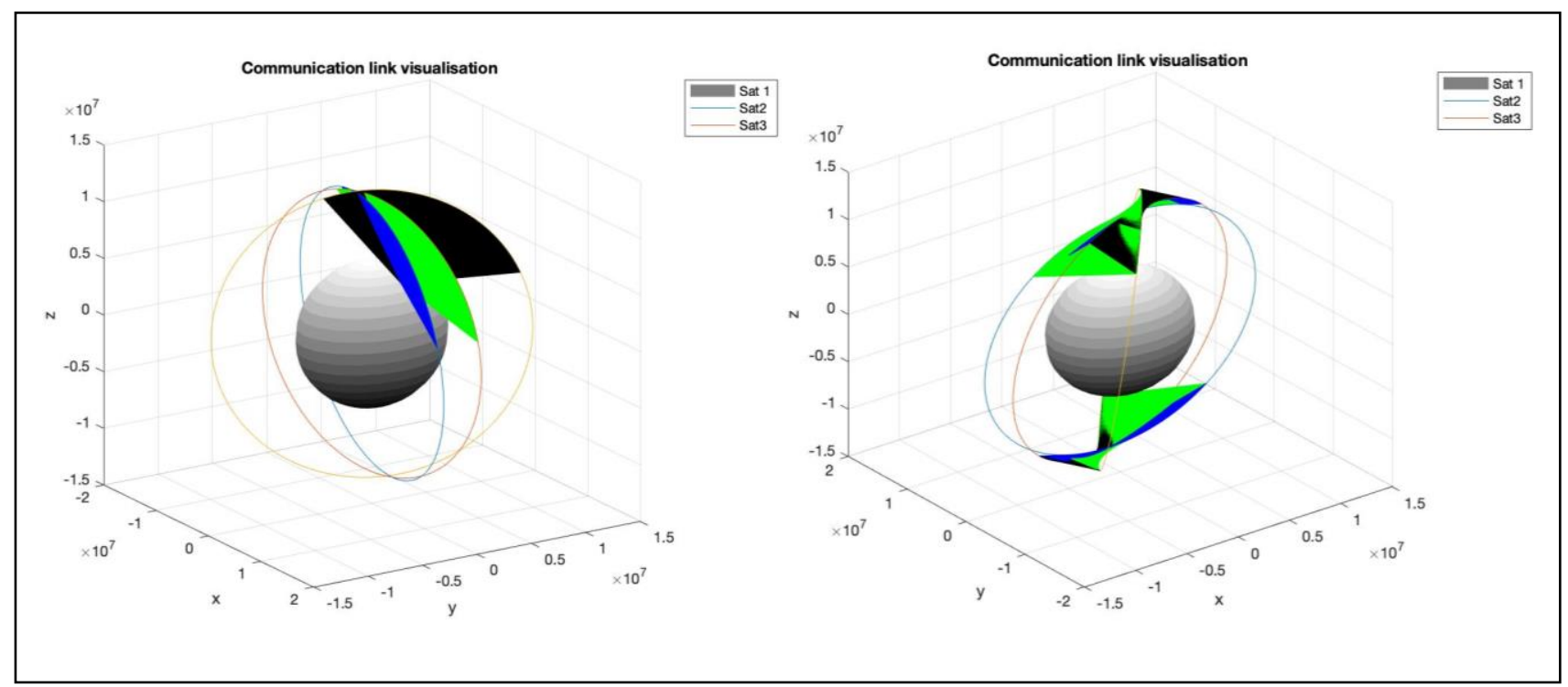

Fig. 5. Satellite position and orientation simulation results

The optimization problem was split between form factors to optimize the computational power required for simulation. Thus, all 6 cases were reviewed separately for different orbital configurations and maximum characteristics for each particular case were defined. Fig. 5 visualizes the map of solutions for the optimization problem for the CubeSat from factor $6 \mathrm{U}$. 
Stepanova, D. \& Pryanichnikov, V.: Requirements Optimization for Small Satellite ...

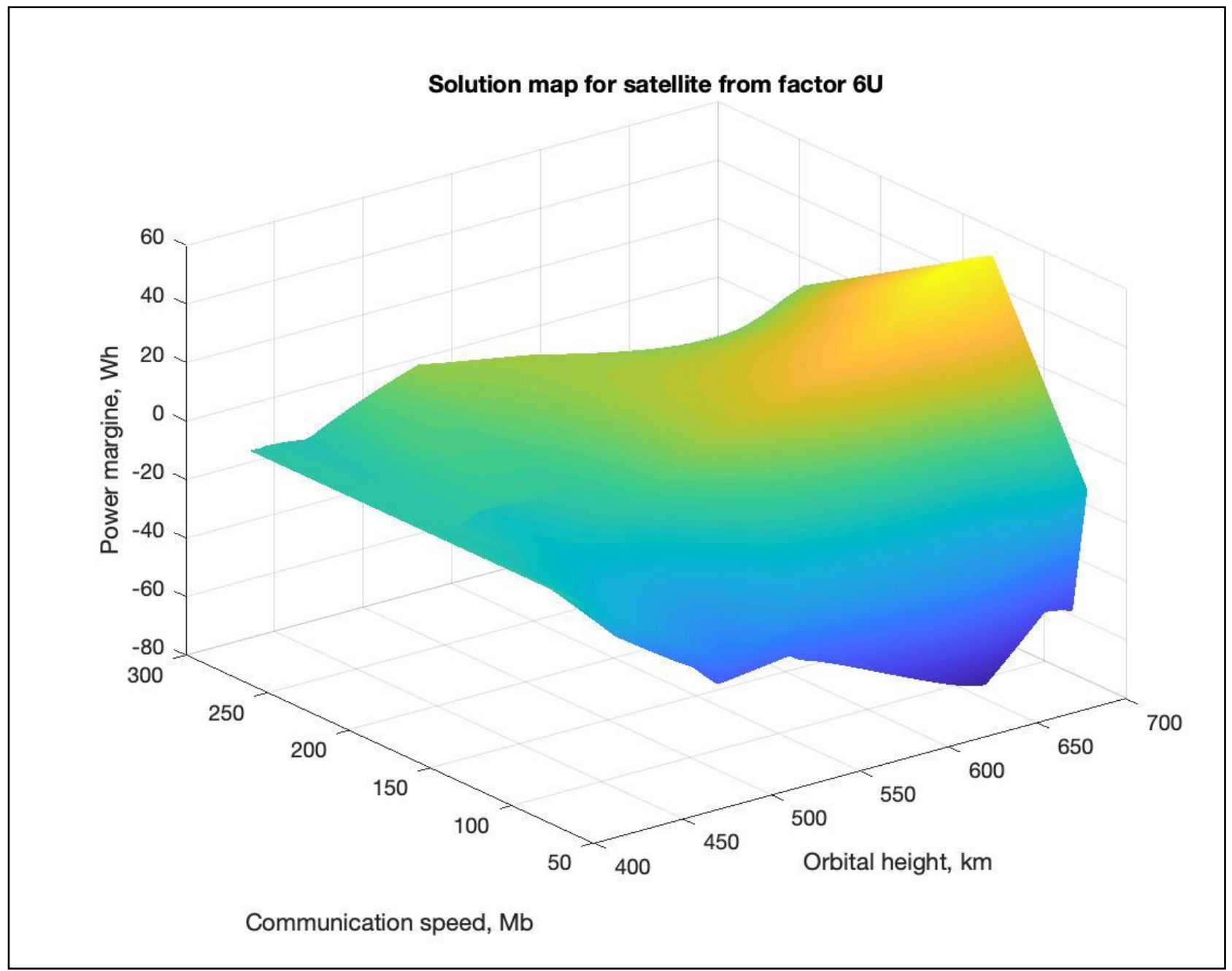

Fig. 6. Satellite solution map for form factor $6 \mathrm{U}$

Based on the solutions set for optimization problem, the average and maximum parameters for the system were defined. Table 1 presents average parameters for intersatellite communication scenario, while Table 2 shows results for satellite to ground station communication case.

\begin{tabular}{|l|l|l|}
\hline $\begin{array}{l}\text { Satellite from } \\
\text { factor }\end{array}$ & $\begin{array}{l}\text { Average power } \\
\text { margin, Wh }\end{array}$ & $\begin{array}{l}\text { Average communication } \\
\text { speed, Mbs }\end{array}$ \\
\hline $1 \mathrm{U}$ & 0.3 & 12 \\
\hline $2 \mathrm{U}$ & 0.7 & 58 \\
\hline $3 \mathrm{U}$ & 1.2 & 243 \\
\hline $6 \mathrm{U}$ & 5.2 & 292 \\
\hline $12 \mathrm{U}$ & 3.9 & 458 \\
\hline $16 \mathrm{U}$ & 4.2 & 682 \\
\hline
\end{tabular}

Tab. 1. Simulation results for intersatellite scenario 


\begin{tabular}{|l|l|l|}
\hline $\begin{array}{l}\text { Satellite from } \\
\text { factor }\end{array}$ & $\begin{array}{l}\text { Average power } \\
\text { margin, Wh }\end{array}$ & $\begin{array}{l}\text { Average communication } \\
\text { speed, Mbs }\end{array}$ \\
\hline $1 \mathrm{U}$ & 0.7 & 8 \\
\hline $2 \mathrm{U}$ & 1.3 & 45 \\
\hline $3 \mathrm{U}$ & 1.8 & 214 \\
\hline $6 \mathrm{U}$ & 5.9 & 287 \\
\hline $12 \mathrm{U}$ & 4.5 & 421 \\
\hline $16 \mathrm{U}$ & 3.9 & 668 \\
\hline
\end{tabular}

Tab. 2. Simulation results for satellite to ground station scenario

As it can be observed, the cases of $1 \mathrm{U}$ and $2 \mathrm{U}$ have a big gap in communication speed with other cases. This is due to the attitude determination system accuracy. The most optimal from the point of view of power margin / communication speed balance is the case of $6 \mathrm{U}$ CubeSat - this satellite for factor balances requirements for the attitude control while maintaining high power generation capabilities. Finally, the bigger the satellite - the easier it is to accommodate more advanced attitude control system and achieve higher pointing capabilities and thus, higher communication speeds.

\section{Conclusion}

The present work has demonstrated the approach to define requirements for a laser communication mission design, including satellite and optical terminal, and find an optimal setup for several scenarios. As the result, the configuration of $6 \mathrm{U}$ CubeSat seems the most attractive from the point of view of balance between communication speed and power requirements, while the conclusion that the bigger the satellite the more communication speed is available shall be made.

Several assumptions have limited the scope of the work. For example, placement of multiple transceivers will complicate the optimisation problem and the operational scenario; however, such configuration might be useful in various missions with intersatellite link requirements: this can provide omnidirectional connectivity and multiple connections at a time. Thus, in future work this case shall be added to the list of constraints. Another limitation to review is an amount of ground stations in the scenario. This parameter might be critical for a number of missions. Besides that, it will also influence optimisation problem considerably.

The present research has showed, that $6 \mathrm{U}$ CubeSat form factor might be the most interesting to review in more detail. Thus, the next step in the research would be define the instrumentation setup for the satellite and continue the analysis of particular CubeSat mission. Adjusting the model for a particular hardware, introducing the sources of errors and noises will be the next steps. Finally, the control system model will finalize the simulation part of the work. On the other hand, the experimental setup for validation of the requirements set shall be developed and its capabilities shall be tested. 
Stepanova, D. \& Pryanichnikov, V.: Requirements Optimization for Small Satellite ...

\section{References}

Kaymak, Y., Rojas-Cessa, R., Feng, J., Ansari, N., Zhou, M., \& Zhang, T. (2018). A survey on acquisition, tracking, and pointing mechanisms for mobile free-space optical communications. IEEE Communications Surveys \& Tutorials, 20(2), 11041123.

Janson, S. W., \& Welle, R. P. (2014, August). The NASA optical communication and sensor demonstration program: an update. In 28th Annual AIAA/USU Conference on Small Satellites (pp. 4-7).

Stepanova, D., Pryanichnikov, V., Khandorin, S., Kuznetsov, A. and Kulchitsky, A., 2018. High-speed image processing technique for pointing and tracking system enabling free-space optical communications. Annals of DAAAM \& Proceedings, 29. Rose, T. S., Rowen, D. W., LaLumondiere, S., Werner, N. I., Linares, R., Faler, A., ... \& Utter, A. (2019, July). Optical communications downlink from a 1.5 U CubeSat: OCSD program. In International Conference on Space Optics-ICSO 2018 (Vol. 11180, p. 111800J). International Society for Optics and Photonics.

Clements, E., Aniceto, R., Barnes, D., Caplan, D., Clark, J., del Portillo, I., ... \& Morgan, R. (2016). Nanosatellite optical downlink experiment: design, simulation, and prototyping. Optical Engineering, 55(11), 111610.

Sst-soa.arc.nasa.gov. (2019). 09. Communications - State of the Art of Small Spacecraft Technology. [online] Available at: https://sst-soa.arc.nasa.gov/09communications [Accessed 12 Oct. 2019].

Iwamoto, K., Araki, T., Kobayashi, Y., Sawada, H., Ito, T., Sano, T., Takeshita, Y., Ota, S., Ueno, M., Shibata, K.I. and Nakao, T., 2017, November. Experimental verifications on small optical inter-satellite communication system for small and micro satellites. In 2017 IEEE International Conference on Space Optical Systems and Applications (ICSOS) (pp. 59-62). IEEE.

Cui, P., \& Yan, N. (2012). Research on modeling of the agile satellite using a single gimbal magnetically suspended CMG and the disturbance feedforward compensation for rotors. Sensors, 12(12), 16964-16987.

Ivanov, D., Ovchinnikov, M., Ivlev, N., \& Karpenko, S. (2015). Analytical study of microsatellite attitude determination algorithms. Acta Astronautica, 116, 339-348.

Abdo, M., Vali, A. R., Toloei, A., \& Arvan, M. R. (2013). Research on the crosscoupling of a two axes gimbal system with dynamic unbalance. International Journal of advanced robotic systems, 10(10), 357.

Li, X., Cheng, W., \& Li, X. (2014). MODELling OF GIMBAL CONTROL MOMENT GYRO AND ANALYSIS OF GIMBAL DISTURBANCE IMPACT. Tehnicki vjesnik/Technical Gazette, 21(6).

Golubev, Yu.F. (2000). Fundamentals of Theoretical Mechanics. Textbook. Moscow State University named after MV Lomonosov.

Pryanichnikov, Valentin, and Daria Stepanova. "Laser communications prototyping for the group of mobile robots." Annals of DAAAM \& Proceedings (2017): 544-551. 